\title{
ARTICLE OPEN \\ A study on light transmittance through red protective shields modified with different window films
}

\author{
Vanida Nimmanon ${ }^{1}$, Praewpat Pachimsawat ${ }^{1}$, Siribang-on Pibooniyom Khovidhunkit ${ }^{1}$, Bhornsawan Thanathornwong ${ }^{2}$ and \\ Thirayost Nimmanon ${ }^{3}$
}

\begin{abstract}
OBJECTIVES/AIMS: This study aimed to improve effectiveness of red protective shields in filtering unwanted light using window films.

MATERIALS AND METHODS: Red protective shields were modified by placing V-Kool (VK), Scotchtint (ST) or Hüper Optik (HP) window films on both sides. Percentage transmittance (\%T) of light with a wavelength of 190-990 nm was determined using a double-beam ultraviolet (UV) and visible spectrophotometer.

RESULTS: In UV light (190-390 nm) and visible light $(430-590 \mathrm{~nm})$ ranges, \%T in all modified groups and the control was below $2.5 \%$. An increase in \%T was observed at the wavelength of $630 \mathrm{~nm}$, when all the modified shields showed superior effectiveness in light filtration over the control. In the infrared spectrum $(700-990 \mathrm{~nm}), \% \mathrm{~T}$ in the control was constantly high, ranging from 86 to $91 \%$, compared to \%T of $2-38 \%$ in all the modified groups, with the application of VK on both sides being the most effective group, followed by a combination of VK and HP.

CONCLUSION: This study has introduced an economical and simple, yet highly effective, means of enhancing the efficiency of a red plastic protection shield in filtering unwanted infrared light, thereby additionally providing protection for dental personnel from potential ocular damages.
\end{abstract}

BDJ OPEN (2017) 3, 17014; doi:10.1038/bdjopen.2017.14; published online 30 June 2017

\section{INTRODUCTION}

Nowadays, the light curing unit is an essential equipment in the dental clinic. It is available in many types, with the quartztungsten-halogen light curing unit being the most commonly used type in the dental office. This instrument employs a tungsten halogen lamp to generate radiation with wavelengths between 350 and 2,500 nm. These wavelengths include ultraviolet (UV), visible light and infrared spectra, with an emission peak between 970 and $980 \mathrm{~nm} .^{1}$ The radiation with wavelengths of less than $380 \mathrm{~nm}$ and more than $520 \mathrm{~nm}$ is undesired and thereby purged, $^{2,3}$ leaving a broad spectral range of light emission for the use in the curing process of a composite resin. ${ }^{4}$ Light at a wavelength of $468 \mathrm{~nm}$ is the most effective in activating composite resins ${ }^{5}$ as well as other light-sensitive dental materials, such as bonding agents, glass ionomer cements and sealants. ${ }^{6}$ However, this blue visible light is not always safe to the eyes, given that it has been reported to cause eye injuries with possible association with development of age-related macular degeneration, ${ }^{7}$ a leading cause of permanent visual loss and blindness in aged people. ${ }^{8}$ Importantly, even though all four types of light curing units tested were not associated with a risk of ocular damages mediated by UV light, potential ocular hazards mediated by blue light were demonstrated upon cumulative eye exposure of the light generated by high-power lamps for only $6 \mathrm{~s}$ at a $30-\mathrm{cm}$ distance over an 8-h working day. ${ }^{3}$ Furthermore, infrared light has been shown to be hazardous to both the lens ${ }^{9}$ and the retina ${ }^{10}$ leading to the suggestion of it being a potential contributory factor of cataract and photothermal retinal injuries, respectively.
Many studies have reported that light curing units not only provide blue visible light, but also emit other unwanted spectra of light. An investigation on radiation emission from nine commercial light curing units showed that only two of them produced radiation at an acceptable wavelength, whereas many of the rest undesirably emitted light with wavelengths above the blue visible light spectrum, and two of these units also radiated light within the infrared spectrum. ${ }^{11}$ Supporting this previous investigation, only three out of five commercial light curing units were demonstrated to emit light within a suitable wavelength range. $^{12}$ According to the threshold limit value of the American Conference of Governmental Industrial Hygienists, the maximum permissible exposure duration in $24 \mathrm{~h}$ was shown in 12 models of curing light units to range from 4 to $110 \mathrm{~min}$, with the highest value resulting from an old unit. ${ }^{13}$ With regard to this finding, cumulative blue light retinal exposure over an 8-h working day as a result of a total of eight 30-s cures would exceed the threshold limit value recommended by the American Conference of Governmental Industrial Hygienists, provided that the light source was positioned where dentists could see the exposed tip. In addition, the authors noted the highly possible occurrence of lamp deterioration and filter cracking as a result of long-termed use of light curing units, suggesting the risk of eye injuries to dentists and their assistants if insufficiently protected. Despite the presence of well-established guidelines for eye safety in operative dentistry, ignorance and thereby suboptimal conformation to these rules among dental personnel have been reported. ${ }^{14}$

In order to provide safety and protection for the eyes from hazardous radiation generated by light curing units, light protective equipment has been developed. There are four major

\footnotetext{
${ }^{1}$ Department of Advanced General Dentistry, Faculty of Dentistry, Mahidol University, Bangkok, Thailand; ${ }^{2}$ Department of General Dentistry, Faculty of Dentistry, Srinakharinwirot University, Bangkok, Thailand and ${ }^{3}$ Department of Pathology, Phramongkutklao College of Medicine, Bangkok, Thailand.

Correspondence: T Nimmanon (thirayost@outlook.com)

Received 1 March 2017; accepted 19 May 2017
} 
Table 1. A comparison of percentage light transmittance $(\% \mathrm{~T})$ of red translucent plastic shields with and without a combination of window films

\begin{tabular}{|c|c|c|c|c|c|c|c|}
\hline Wavelength $(\mathrm{nm})$ & No film & $2 V K$ & $2 S T$ & $2 H P$ & $V K+S T$ & $S T+H P$ & $V K+H P$ \\
\hline 190 & $1.70 \pm 0.76$ & $1.39 \pm 0.38$ & $1.42 \pm 0.79$ & $1.50 \pm 0.74$ & $1.47 \pm 0.65$ & $1.26 \pm 0.35$ & $1.52 \pm 0.77$ \\
\hline 230 & $1.72 \pm 0.73$ & $1.41 \pm 0.38$ & $1.43 \pm 0.77$ & $1.51 \pm 0.72$ & $1.50 \pm 0.66$ & $1.27 \pm 0.37$ & $1.53 \pm 0.77$ \\
\hline 270 & $1.70 \pm 0.72$ & $1.39 \pm 0.37$ & $1.42 \pm 0.77$ & $1.49 \pm 0.69$ & $1.47 \pm 0.64$ & $1.27 \pm 0.36$ & $1.51 \pm 0.76$ \\
\hline 310 & $1.55 \pm 0.69$ & $1.28 \pm 0.33$ & $1.30 \pm 0.73$ & $1.37 \pm 0.67$ & $1.35 \pm 0.59$ & $1.16 \pm 0.33$ & $1.40 \pm 0.72$ \\
\hline 350 & $2.48 \pm 0.88$ & $2.12 \pm 0.46$ & $2.14 \pm 0.91$ & $2.23 \pm 0.85$ & $2.21 \pm 0.77$ & $1.96 \pm 0.46$ & $2.25 \pm 0.91$ \\
\hline 390 & $1.84 \pm 0.68$ & $1.55 \pm 0.36$ & $1.57 \pm 0.72$ & $1.65 \pm 0.66$ & $1.63 \pm 0.60$ & $1.43 \pm 0.35$ & $1.66 \pm 0.70$ \\
\hline 430 & $1.76 \pm 0.69$ & $1.47 \pm 0.35$ & $1.50 \pm 0.71$ & $1.59 \pm 0.65$ & $1.55 \pm 0.61$ & $1.36 \pm 0.35$ & $1.60 \pm 0.69$ \\
\hline 470 & $1.69 \pm 0.66$ & $1.42 \pm 0.35$ & $1.45 \pm 0.70$ & $1.52 \pm 0.64$ & $1.49 \pm 0.59$ & $1.30 \pm 0.35$ & $1.53 \pm 0.68$ \\
\hline 510 & $1.60 \pm 0.64$ & $1.33 \pm 0.33$ & $1.36 \pm 0.68$ & $1.44 \pm 0.61$ & $1.41 \pm 0.57$ & $1.22 \pm 0.32$ & $1.45 \pm 0.66$ \\
\hline 550 & $1.50 \pm 0.64$ & $1.24 \pm 0.32$ & $1.27 \pm 0.68$ & $1.34 \pm 0.61$ & $1.31 \pm 0.56$ & $1.13 \pm 0.32$ & $1.35 \pm 0.65$ \\
\hline 590 & $1.40 \pm 0.61$ & $1.12 \pm 0.30$ & $1.15 \pm 0.66$ & $1.22 \pm 0.58$ & $1.19 \pm 0.54$ & $1.01 \pm 0.30$ & $1.23 \pm 0.63$ \\
\hline 630 & $67.27 \pm 3.26$ & $36.97 \pm 2.25$ & $32.41 \pm 2.27$ & $27.34 \pm 1.60$ & $33.88 \pm 2.10$ & $28.62 \pm 1.94$ & $31.92 \pm 1.85$ \\
\hline 670 & $82.69 \pm 3.12$ & $39.15 \pm 2.01$ & $37.12 \pm 2.40$ & $31.72 \pm 1.65$ & $38.51 \pm 2.24$ & $34.39 \pm 2.00$ & $34.70 \pm 1.78$ \\
\hline 710 & $85.85 \pm 2.37$ & $32.24 \pm 1.96$ & $37.06 \pm 2.75$ & $29.42 \pm 1.18$ & $33.92 \pm 2.66$ & $32.63 \pm 2.24$ & $30.57 \pm 1.70$ \\
\hline 750 & $87.89 \pm 1.49$ & $24.93 \pm 1.57$ & $37.47 \pm 3.85$ & $29.87 \pm 2.29$ & $30.54 \pm 2.53$ & $32.50 \pm 3.00$ & $27.61 \pm 1.73$ \\
\hline 790 & $89.88 \pm 0.65$ & $15.92 \pm 1.30$ & $38.42 \pm 3.47$ & $25.31 \pm 1.74$ & $24.27 \pm 3.11$ & $32.17 \pm 3.29$ & $19.48 \pm 1.76$ \\
\hline 830 & $91.47 \pm 1.23$ & $10.53 \pm 0.82$ & $36.42 \pm 3.63$ & $26.49 \pm 2.94$ & $19.09 \pm 2.12$ & $28.18 \pm 3.75$ & $16.73 \pm 1.63$ \\
\hline 870 & $90.80 \pm 0.85$ & $6.65 \pm 0.53$ & $34.55 \pm 4.62$ & $18.82 \pm 2.12$ & $15.19 \pm 1.79$ & $26.33 \pm 3.40$ & $10.39 \pm 1.05$ \\
\hline 910 & $88.75 \pm 1.01$ & $3.95 \pm 0.52$ & $30.77 \pm 3.44$ & $19.61 \pm 2.62$ & $10.92 \pm 1.49$ & $24.78 \pm 3.15$ & $8.83 \pm 1.42$ \\
\hline 950 & $88.82 \pm 2.33$ & $2.65 \pm 0.42$ & $31.73 \pm 2.46$ & $12.28 \pm 1.04$ & $8.31 \pm 1.19$ & $19.65 \pm 1.52$ & $5.17 \pm 0.77$ \\
\hline 990 & $87.29 \pm 2.64$ & $1.97 \pm 0.24$ & $28.92 \pm 2.13$ & $12.62 \pm 1.86$ & $6.67 \pm 0.95$ & $20.20 \pm 1.95$ & $4.69 \pm 0.77$ \\
\hline
\end{tabular}

Abbreviations: HP, the Hüper Optik film; ST, the Scotchtint film; VK, the V-Kool film.

types of protective shields, including safety glasses, caps of light conductor tips or skirt shields, unit-attached eye shields, and unitindependent eye shields or separated shields. ${ }^{15}$ Protective shields are usually made of orange glasses, ${ }^{16}$ given that orange and blue are complementary colours, and as such, the orange colour efficiently absorbs blue visible light. Supporting this, orange protective shields were confirmed to have superior capability to eliminate blue visible light when compared to red protective shields. ${ }^{12}$ However, one study proposed the use of yellow or red protective shields, instead of orange ones, to prevent eye hazards. ${ }^{17}$ Our group have previously examined light transmittance through orange unit-independent protective shields, showing $2 \%$ light transmittance in the wavelength range of 200-500 nm (UV and visible light) and 80-90\% light transmittance in the wavelength range of more than $500 \mathrm{~nm}$, suggesting partial filtering efficiency of the shields for light with higher wavelengths. ${ }^{18}$ Importantly, supporting the use of red protective shields, our group have also investigated percentage light transmittance of locally made plastic shields of different colours, revealing that red shields more effectively filtered radiation than orange shields did, even though neither of them was able to filter infrared light. ${ }^{19}$

Given that none of the commercially available protective shields can provide complete filtration of unwanted light while allowing optimal operation view, this study aimed to improve filtration effectiveness of the shields by modifying them using window films. Red protective shields were covered on both sides with a combination of three types of window films: V-Kool (VK), Scotchtint (ST) and Hüper Optik (HP). Percentage transmittance $(\% \mathrm{~T})$ of light in the wavelength range of 190-990 nm was determined using a double-beam UV and visible spectrophotometer.

\section{MATERIALS AND METHODS}

Red translucent plastic plates (Thai Poly Acrylic Corp, Bangkok, Thailand), $3 \mathrm{~mm}$ in thickness, were cut into 210 rectangular pieces with a dimension of $2 \mathrm{~cm} \times 1.3 \mathrm{~cm}$ using an electric cutting machine. These pieces of plastic shields were covered with a combination of three different types of window films, consisting of VK Vicole 70 (Southwall Technologies, Palo Alto, CA, USA), Scothtint RE 65NIARL (ST, 3M Solar Optical Products, St Paul, Minnesota, MN, USA) and Hüper Optik HP Sech (Southwall
Technologies). They were divided into seven groups, including the control without modification, VK on both sides (2VK), ST on both sides (2ST), HP on both sides (2HP), a combination of VK and ST (VK+ST), a combination of ST and $\mathrm{HP}(\mathrm{ST}+\mathrm{HP})$ and a combination of VK and HP (VK+HP). All groups were evaluated for \%T at different wavelengths from 190 to $990 \mathrm{~nm}$ with $40 \mathrm{~nm}$ increments using a double-beam UV and visible spectrophotometer (Perkin-Elmer UV and Visible Spectrometer, Lambda 14). Each specimen was tested twice, and \%T was shown as mean values. Statistical analysis was performed using ANOVA and Scheffe multiple comparison tests. Significance was assumed when $P<0.05$.

\section{RESULTS}

\%T was determined for red plastic shields with or without modifications at wavelengths between 190 and $990 \mathrm{~nm}$. All data are shown in Table 1 and Figure 1. In the range of either UV light $(190-390 \mathrm{~nm})$ or visible light $(430-590 \mathrm{~nm})$, all groups where shown to have less than $2.5 \%$ light transmittance with no significant difference observed between the shields with and without modifications. In the range of $590-670 \mathrm{~nm}$, light transmittance in the control group was dramatically increased to $67 \%$ and $83 \%$ at the wavelengths of $630 \mathrm{~nm}$ and $670 \mathrm{~nm}$, respectively. In contrast, light transmittance in all the modified groups was increased to the maximum of $40 \%$ at both these wavelengths, which was significantly less than the control. Among the modified groups, 2HP was shown to have the lowest light transmittance in this wavelength range $(630-670 \mathrm{~nm})$, followed by $\mathrm{ST}+\mathrm{HP}, 2 \mathrm{ST}, \mathrm{VK}+\mathrm{HP}, \mathrm{VK}+\mathrm{ST}$ and $2 \mathrm{VK}$, respectively.

When the wavelengths reached the infrared range $(710 \mathrm{~nm})$, light transmittance in the control group remained constantly high, ranging from 86 to $91 \%$, with a slight increase between 710 and $830 \mathrm{~nm}$ and a slight decrease between 830 and $990 \mathrm{~nm}$. On the contrary, light transmittance in all the modified groups was gradually decreased until the wavelength of $990 \mathrm{~nm}$ and was shown to be significantly less than the control group throughout the range of 710-990 nm, with the 2ST group and the 2VK group being shown to have the highest and the lowest transmittance across the modified groups, respectively. The differences in light transmittance among the modified groups became more clearly evident at higher wavelengths, particularly those between 870 and $990 \mathrm{~nm}$. In this extreme wavelength range of $870-990 \mathrm{~nm}$, the lowest light transmittance was demonstrated in the 2VK group, 


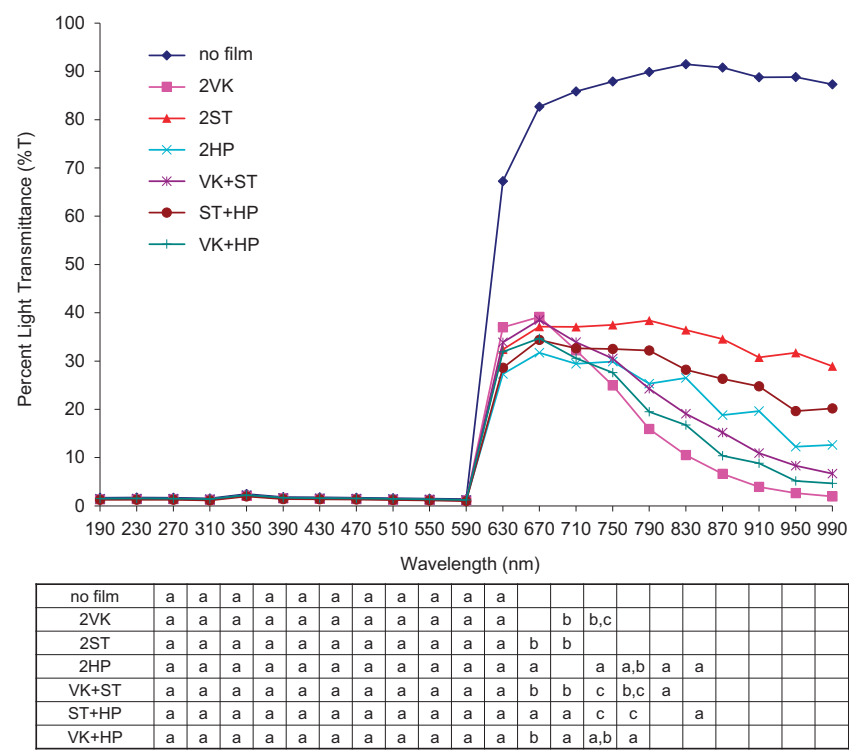

Figure 1. A comparison of percentage light transmittance of red translucent plastic shields with and without applications of different window films: VK, ST and HP at different wavelengths. The table demonstrates statistical analysis comparing between groups. The groups that are indicated by the same letters $(a-c)$ at a wavelength are not statistically different $(P>0.05)$ from each other.

followed by $\mathrm{VK}+\mathrm{HP}, 2 \mathrm{HP}, \mathrm{VK}+\mathrm{ST}, \mathrm{ST}+\mathrm{HP}$ and $2 \mathrm{ST}$ groups, respectively.

\section{DISCUSSION}

Most studies on ocular damages caused by light generated by light curing units have focused on UV light ${ }^{20}$ and visible light, ${ }^{7}$ since these wavelength spectra are known for their causative relationship with retinal injuries. ${ }^{21,22}$ Accordingly, protective equipment has been designed to filter these light spectra to decrease exposure of dentistry personnel to these undesired wavelengths of light. An investigation demonstrated that all four types of light curing units, consisting of plasma arc, low-power light-emitting diode, high-power light-emitting diode and quartz-tungsten-halogen, radiated a negligible amount of effective UV light under all curing conditions and thereby did not increase the risk of UV light-induced ocular damages. ${ }^{3}$ Furthermore, many investigations have shown the effectiveness of protective shields in filtering UV light. ${ }^{12,18,19}$ Confirming this, we have shown in this present study that red plastic protective shields were able to filter UV light up to $98 \%$, allowing only $2 \%$ of it transmitting through the shields. The highly efficient filtering capacity of these shields can be at least partly attributed to intrinsic properties of plastic material, which contains chemical bonds that absorb the energy of the radiation at a lower wavelength.

In contrast to the ability of the shield to filter UV light, the shields without modification was shown to be less effective in preventing transmittance of visible light $(400-700 \mathrm{~nm})$, which is composed of seven colours (violet, purple, blue, green, yellow, orange and red). ${ }^{6,11}$ Given that red is a colour complementary to green, this colour is theoretically highly effective in filtering green light but less effective in filtering red light. Noteworthy, our group have reported a superior filtering ability of red plastic shields over orange plastic shields, which are commonly used in dental practice. ${ }^{19}$ Importantly, the modifications of the shields using window films were shown to significantly reduce percentage transmittance of light in this visible range by at least 55\%, thereby adding further protection against visible light-related eye injuries.

In contrast to UV light and visible light, the relationship between infrared light $(700-990 \mathrm{~nm})$ and ocular injuries is often overlooked. For example, a recent investigation evaluated protective filters against radiation specifically within the wavelength range of $400-525 \mathrm{~nm},{ }^{23}$ which belongs to the visible light spectrum. Nevertheless, biohazards of this infrared light has also been occasionally reported. One study demonstrated that proteins in the eye lens is notably sensitive to infrared radiation, suggesting the relationship between this light and development of cataract. ${ }^{9}$ Moreover, infrared light can also induce photomechanical, photothermal and photochemical damages to the retina, ${ }^{10}$ thereby posing a risk to visual impairment. In this present study, we considered a wider range of light, also including the infrared spectrum in the investigation. In contrast to the unmodified red plastic protective shields, which allowed up to $91 \%$ of infrared light to transmit through them, the modifications with window films were able to significantly reduce the transmittance of infrared light by $77-98 \%$ when compared to the unmodified shields.

Among all the three types of window films tested in this present study, the VK film was shown to be the most effective in light filtration. This VK film allowed some transmission of light within the harmless visible light spectrum, thereby providing adequate visibility for the dentist during an operation. Consistent with our previous investigation, ${ }^{19}$ up to $98 \%$ of infrared light was filtered when the VK film was applied on both sides of the shields. Furthermore, a combination of the VK film with another type of window films was shown to enhance light filtration effectiveness of the other window film compared to when it was used without the VK film, suggesting the complementary effect of the VK film to other film types. The infrared light filtrating capability of the VK film can be attributed to the silver particle in the metallic layer of the film, which has higher reflective properties than aluminium, nickel and copper, which are contained in the Scotchtinct film. ${ }^{24}$ It is not clear why the HP had less infrared light filtering capability than the VK film regardless of it also containing the silver particle in its metallic layer.

\section{CONCLUSION}

There have been pieces of evidence showing that some light curing units that are generally used nowadays may emit some light in an inappropriate wavelength range, ${ }^{11,12}$ which could be hazardous to the eyes. We have demonstrated that unmodified plastic shields may not be able to provide adequate filtering for this undesired light, particularly that which is in the infrared spectrum. We therefore would like to recommend the application of window films, particularly the VK film, to the conventional protective shields in order to increase the filtration efficiency of the shields. This will further provide extra protection for the dentistry personnel from any light-induced ocular damages while allowing them to have sufficient visualisation during the operation.

\section{ACKNOWLEDGEMENTS}

We express our gratitude to Pattana Rakkwamsuk for his invaluable suggestions on the window films, Tharadol Kengganpanich for his kind advice regarding statistical analysis and Sombat Kongwithaya for his help with laboratory techniques.

\section{COMPETING INTERESTS}

The authors declare no conflict of interest. 


\section{REFERENCES}

1 Elvidge CD, Keith DM, Tuttle BT, Baugh KE. Spectral identification of lighting type and character. Sensors 2010; 10: 3961-3988.

2 Burgess JO, Walker RS, Porche CJ, Rappold AJ. Light curing--an update. Compend Contin Educ Dent 2002; 23: 889-892.

3 Labrie D, Moe J, Price RBT, Young ME, Felix CM. Evaluation of ocular hazards from 4 types of curing lights. J Can Dent Assoc 2011; 77: b116.

4 Price RB, Ferracane JL, Shortall AC. Light-curing units: a review of what we need to know. J Dent Res 2015; 94: 1179-1186.

5 Ferreira APB, Soares Júnior PC, Souza EM, Rached RN, Pezzin SH, Vieira S. Wavelength of experimental LEDS: hardness, elastic modulus, degree of conversion and temperature rise of a microhybrid composite. Mater Res 2015; 18: 240-244.

6 Shortall A, Harrington E. Guidelines for the selection, use, and maintenance of visible light activation units. Br Dent $J$ 1996; 181: 383-387.

7 Taylor HR, West S, Muñoz B, Rosenthal FS, Bressler SB, Bressler NM. The long-term effects of visible light on the eye. Arch Ophthalmol 1992; 110: 99-104.

8 Bourne RRA, Jonas JB, Flaxman SR, Keeffe J, Leasher J, Naidoo K et al. Prevalence and causes of vision loss in high-income countries and in Eastern and Central Europe: 1990-2010. Br J Ophthalmol 2014; 98: 629-638.

9 Aly E, Mohamed E. Effect of infrared radiation on the lens. Indian J Ophthalmol 2011; 59: 97.

10 Youssef PN, Sheibani N, Albert DM. Retinal light toxicity. Eye 2011; 25: 1-14.

11 Davis LG, Baker WT, Cox EA, Marshall J, Moseley TJ. Optical hazards of blue light curing units: preliminary results. Br Dent J 1985; 159: 259-262.

12 Ellingson OL, Landry RJ, Bostrom RG. An evaluation of optical radiation emissions from dental visible photopolymerization devices. J Am Dent Assoc 1986; 112: 67-70.

13 Eriksen P, Moscato PM, Franks JK, Sliney DH. Optical hazard evaluation of dental curing lights. Community Dent Oral Epidemiol 1987; 15: 197-201.

14 Farrier SL, Farrier JN, Gilmour ASM. Eye safety in operative dentistry-a study in general dental practice. Br Dent J 2006; 200: 218-223.

15 Piché J, Belanger M. Potential damaging effects of blue light on the eye. Oral Health 1996; 86: 43-46.

16 Walsh LJ, Brostek AM. Minimum intervention dentistry principles and objectives. Aust Dent J 2013; 58(Suppl 1): 3-16.

17 Pollack BF, Lewis AL. Visible light resin-curing generators: a comparison. Gen Dent 1981; 29: 488-493.

18 Pachimsawat P, Nimmanon V, Jitkitsadakul P. The light transmittance of three different protective shields: a comparative study. J Dent Assoc Thai 1998; 48: 275-287.

19 Nimmanon V, Tovivich P, Pachimsawat P, Kanchanavasita W, Thanathornwong P. The light transmittance of modified light protective shields: a comparative study. J Dent Assoc Thai 2006; 56: 11-29.

20 Nomoto R, McCabe JF, Hirano S. Comparison of halogen, plasma and LED curing units. Oper Dent 29: 287-294.

21 Sliney DH, Hunter JJ, Delori FC, Williams DR, Mellerio J. Competing photochemical retinal damage mechanisms from visible light: implications for human retinal exposure limits. Invest Ophthalmol Vis Sci 2010; 51: 3456.

22 Glickman RD. Ultraviolet phototoxicity to the retina. Eye Contact Lens 2011; 37: 196-205.

23 Bruzell EM, Johnsen B, Aalerud TN, Christensen T. Evaluation of eye protection filters for use with dental curing and bleaching lamps. J Occup Environ Hyg 2007; 4: 432-439.

24 Willey RR. Typical Equipment for Optical Coating Production. In Willey RR (ed), Practical Design and Production of Optical Thin Films, 1st edn New York: Dekker, 1996, pp 141-190.

(i) This work is licensed under a Creative Commons Attribution 4.0 International License. The images or other third party material in this article are included in the article's Creative Commons license, unless indicated otherwise in the credit line; if the material is not included under the Creative Commons license, users will need to obtain permission from the license holder to reproduce the material. To view a copy of this license, visit http://creativecommons.org/licenses/ by/4.0/

(c) The Author(s) 2017 